\title{
SPECTROSCOPY, INTERACTION WITH RADIATION
}

\section{On the Luminescence of Freshly Introduced $a$-Screw Dislocations in Low-Resistance GaN}

\author{
O. S. Medvedev*, O. F. Vyvenko, and A. S. Bondarenko \\ Saint Petersburg State University, Universitetskaya nab. 7/9, St. Petersburg, 199034 Russia \\ *e-mail: o.s.medvedev@spbu.ru \\ Submitted February 25, 2015; accepted for publication March 3, 2015
}

\begin{abstract}
Using scanning electron microscopy in the cathodoluminescence mode, it is shown that straight segments of $a$-screw dislocations introduced by plastic deformation at room temperature into unintentionally doped low-resistance gallium nitride luminesce in the spectral range 3.1-3.2 eV at $70 \mathrm{~K}$. The spectral composition of dislocation luminescence shows a fine doublet structure with a component width of $\sim 15 \mathrm{meV}$ and splitting of $\sim 30 \mathrm{meV}$, accompanied by LO-phonon replicas. Luminescent screw dislocations move upon exposure to an electron beam and at low temperatures, but retain immobility for a long time without external excitation. Optical transitions involving the quantum-well states of a stacking fault in a split-dislocation core are considered to be the most probable mechanism of the observed phenomenon.
\end{abstract}

DOI: $10.1134 / \mathrm{S} 1063782615090213$

\section{INTRODUCTION}

Dislocation luminescence (DL), i.e., the formation of new luminescence bands with energies lower than the band gap, caused by dislocations, is well known and has been studied in detail for many semiconductors. In germanium and silicon, DL exhibits a large set of spectral lines; for some of them, it was found that their position and fine structure depend on the atomic structure of the cores of split 60-degree dislocations [1, 2]. In direct-gap II-VI binary compounds, only screw dislocations luminesce $[3,4]$; in silicon carbide, opposite partial 30-degree dislocations bounding stacking faults (SFs) luminesce with significantly different DL wavelengths [5].

Gallium nitride is a material widely used as the active element in semiconductor radiation sources of the visible and ultraviolet ranges. As a rule, the density of grown-in dislocations in the most perfect $\mathrm{GaN}$ crystals is no less than $10^{5} \mathrm{~cm}^{-2}$, which makes it particularly important to understand the mechanisms of the effect of dislocations on the electronic properties of this material. However, despite a certain number of studies in this field, the present level of understanding of the electronic properties of dislocations in $\mathrm{GaN}$ is far less than in the above-listed materials.

Experimental data on the effect of dislocations on GaN luminescence, obtained until recently revealed both the nonradiative nature of recombination at dislocations and the appearance of new spectral luminescence bands [6-10].

For grown-in dislocations, luminescence lines were reported at 3.21 [11] and $3.4 \mathrm{eV}$ [6]. For arrays of dislocations introduced by plastic deformation at ele- vated temperatures, emission lines at 1.8, 1.9, 2.4 [12], and $2.9 \mathrm{eV} \mathrm{[13]} \mathrm{were} \mathrm{detected.}$

Recently, we reported on a new DL band in the spectral range of 3.0-3.2 at $70 \mathrm{~K}$ from individual $a$-screw dislocations [14] introduced at room temperature into undoped gallium nitride, detected by the cathodoluminescence (CL) method. Quite recently, DL observation from individual screw dislocations introduced under the same conditions, but into semi-insulating $\mathrm{GaN}$, whose emission energy appeared higher, i.e., $3.35 \mathrm{eV}$ at $4 \mathrm{~K}$, was reported [15]. In this case, according to transmission electron microscopy (TEM) data [15], the dislocations in the studied samples were perfect, i.e., they did not undergo the dissociation characteristic of all glide dislocations in tetrahedrally coordinated semiconductors.

In this paper, we present a detailed description of the properties of the DL band of $a$-screw dislocations in low-resistance gallium nitride, and give arguments in favor of the fact that the cause of the difference in its spectral position from that of the DL in a high-resistance material is associated with the different structures of cores in semiconductors with different Fermilevel positions.

\section{EXPERIMENTAL}

GaN crystals $200-400 \mu \mathrm{m}$ thick grown by the two stage hydride vaporphase epitaxy (HVPE) method at growth temperatures between 1000 and $1150^{\circ} \mathrm{C}$ on sapphire [16] were studied. The grown-in dislocation density was $\sim 10^{6} \mathrm{~cm}^{-2}$. The concentration of uncompensated donors was determined by the capacitance- 

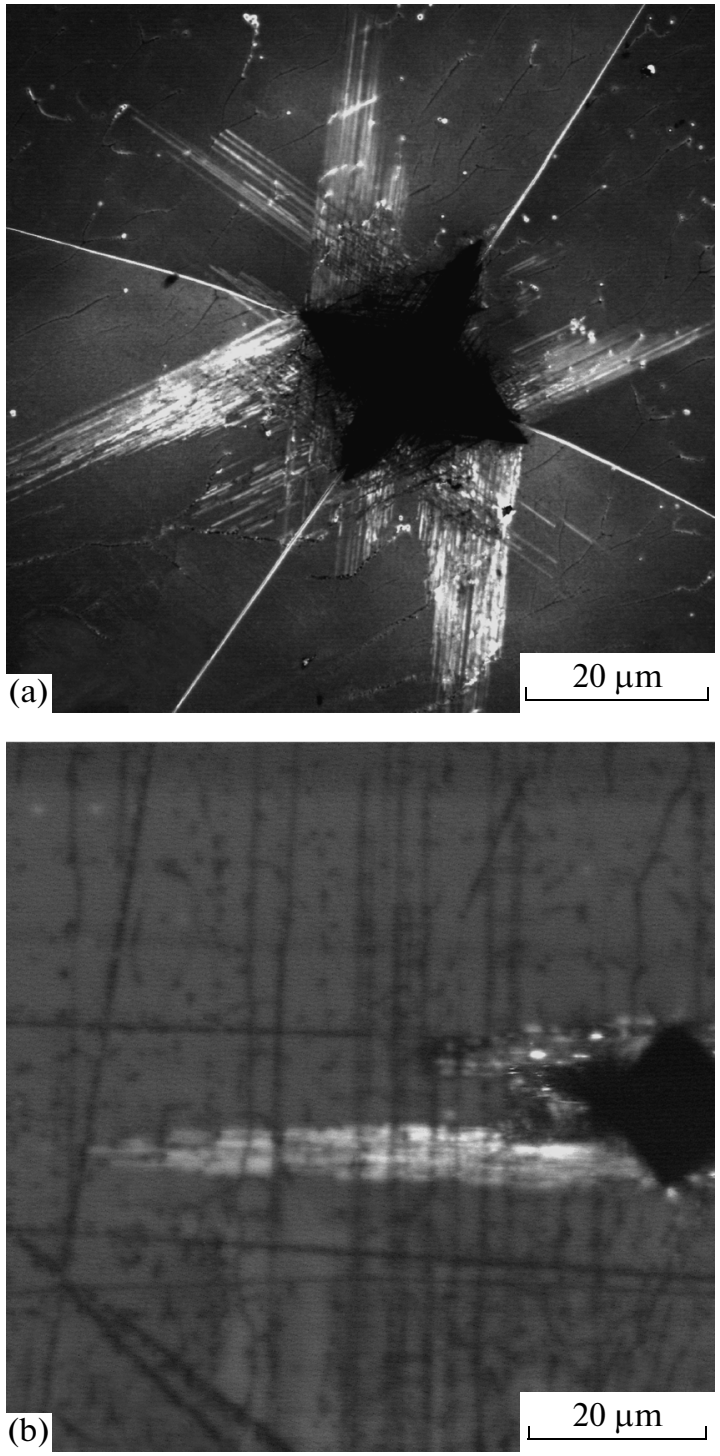

Fig. 1. (a) Cathodoluminescence image in the panchromatic mode, $V_{a}=5 \mathrm{kV}, I_{\text {beam }}=0.5 \mathrm{nA}$, and $T=70 \mathrm{~K}$.

Bright screw dislocations are seen in the $\pm\langle 2 \overline{11} 0\rangle$ directions in the $c(0001)$ plane; dislocation luminescence is dominant over interband luminescence; bright dots correspond to threading dislocations. (b) CL map near the indent on the prismatic surface $(10 \overline{1} 0), V_{a}=15 \mathrm{kV}$, $I_{\text {beam }}=0.5 \mathrm{nA}, T=70 \mathrm{~K}$.

voltage characteristics of Schottky diodes fabricated by gold deposition on the growth surface as $\sim 10^{16} \mathrm{~cm}^{-3}$.

Fresh dislocations were introduced at room temperature by indenting the growth (0001) and prismatic $(10 \overline{1} 0)$ surfaces of gallium nitride with a tetrahedral pyramidal indenter using a Buehler Micromet 5103 hardness tester with loads of $0.1,1$, and $5 \mathrm{~N}$ for $10 \mathrm{~s}$ or by scratching. Dislocation luminescence was also observed in $\mathrm{GaN}$ samples grown by the metalorganic chemical vapor deposition (MOCVD) technology.
Cathodoluminescence studies were performed using a Carl Zeiss Supra 40VP scanning electron microscope equipped with a MonoCL3 system including a parabolic-mirror light collector, a monochromator, and Hamamatsu photomultiplier cooled by a Peltier element. CL radiation was measured in the panchromatic mode in the spectral range of 300-900 $\mathrm{nm}$. The CL measurements were performed at accelerating voltages of $V_{a}$ from 3 to $30 \mathrm{kV}$ at a beam current $I_{\text {beam }}$ from 0.5 to $10 \mathrm{nA}$ and temperatures of $T=70-300 \mathrm{~K}$.

\section{EXPERIMENTAL RESULTS}

Figure 1a shows a map of the CL intensity distribution obtained in the panchromatic mode on the growth surface of the gallium-nitride sample near an indent made with a load of $5 \mathrm{~N}$.

Luminous rays of the dislocation rosette consisting of straight segments extended in three possible directions $\pm\langle 2 \overline{11} 0\rangle$ propagating from the indenter to tens of micrometers are clearly seen. According to the TEM data $[9,13]$, these luminous lines are screw dislocations in small half-loops with head edge dislocations in the prismatic and basal slip systems with the Burgers vector $(a / 3)\langle 2 \overline{11} 0\rangle$.

We note that the prevalence of four of six rosette rays possible for this surface orientation is caused by the features of mechanical stresses near the tetrahedral indenter. Furthermore, in Fig. 1a, we can see irregularly distributed bright dots caused by the amplification of CL collection in the main emission band at points where there are dips in growth near threadingdislocation emergence. A detailed description of the results of the study of grown-in dislocation CL is beyond the present report and will be published additionally.

In the case of monochromatic measurements, freshly introduced screw dislocations in the exciton CL band had dark contrast as reported previously $[8,9,17]$; in the panchromatic mode, the bright contrast of screw dislocations reached $20-30 \%$ at an accelerating voltage of $V_{a}=5 \mathrm{kV}$. We note that images of deeper curvilinear dislocation loops appeared in the CL maps near the growth surface indents at high accelerating voltages, which were seen as dark lines with a contrast of $2.5-5 \%$ in all modes in accordance with previously published data [18].

Straight luminous dislocations propagating in the [1 1210$]$ directions were also detected after indenting the prismatic surfaces and near fresh scratches. Figure $1 \mathrm{~b}$ shows the panchromatic CL map near an indent on the prismatic polished (10 $\overline{1} 0)$ surface, where dark lines along the [0001] axis and dark dots corresponding to grown-in dislocations, which exhibit the properties of nonradiative recombination centers, are also seen. 
Thus, the presented data suggest that straight segments of screw dislocations incorporated into halfloops in both the basal and prismatic slip planes are DL sources.

Local spectral measurements revealed that CL-intensity enhancement at screw dislocations is caused by the appearance of a new spectral emission band of gallium nitride. Figure 2a shows the sample spectra measured at $T=70 \mathrm{~K}$ at fixed electron-beam positions far from (dashed curve) and directly on fresh $a$-screw dislocations (solid curve). The spectrum of dislocation-free regions (Fig. 2, dashed curve) for all samples under study consisted of a free exciton (FE) emission band with an energy of 3.46-3.47 eV, which was dominant in intensity, and two low-intensity bands with maxima near 3.4 and $3.3 \mathrm{eV}$, whereas DL is characterized by the appearance of the intrinsic band in the crystalemission spectrum $(3.1-3.2 \mathrm{eV})$ consisting of two incompletely resolved narrow $\mathrm{DL}_{L}$ and $\mathrm{DL}_{H}$ peaks with phonon replicas (phonon LO) for each. It should be emphasized that the DL intensity at $70 \mathrm{~K}$ in the region with a high density of $a$-screw dislocations was higher than the FE intensity by an order of magnitude, and also exceeded the FE intensity in the dislocationfree region, as shown in the spectra (Fig. 2a). At room temperature, the DL-band spectral position was redshifted according to the temperature dependence of the $\mathrm{GaN}$ band gap.

When recording the DL spectra of single luminous lines, the doublet structure became well resolvable (see Fig. 2b). The FWHM of the composing peaks was $\sim 15 \mathrm{meV}$ which also corresponded to the width of the main peak of exciton luminescence of the crystal. The distance between the $\mathrm{DL}_{L}$ and $\mathrm{DL}_{H}$ doublet components was $\sim 30 \mathrm{meV}$ and was independent of the excitation region within determination error, while the whole doublet was red-shifted along with the main exciton peak as the electron beam receded from the dislocation introduction region. Such a shift in the spectral position of the exciton peak indicates a decrease in macroscopic strains with distance from the indent. The DL red-shift with distance from the indent is shown in two maps of the monochromatic DL distribution for measurements of photon energies of 3.15 and $3.195 \mathrm{eV}$ (see Fig. 3). We can see that luminous dislocations in rosette rays are traced at significantly greater distances at lower detected photon energies.

When the beam was positioned near the emergence of fresh prismatic edge dislocations to the surface and on dislocation half-loops in the basal plane, no additional CL spectral bands were detected.

\section{RESULTS AND DISCUSSION}

In the previous section, we showed that $a$-screw dislocations introduced by plastic deformation at room temperature by $\mathrm{GaN}$ indentation or scratching
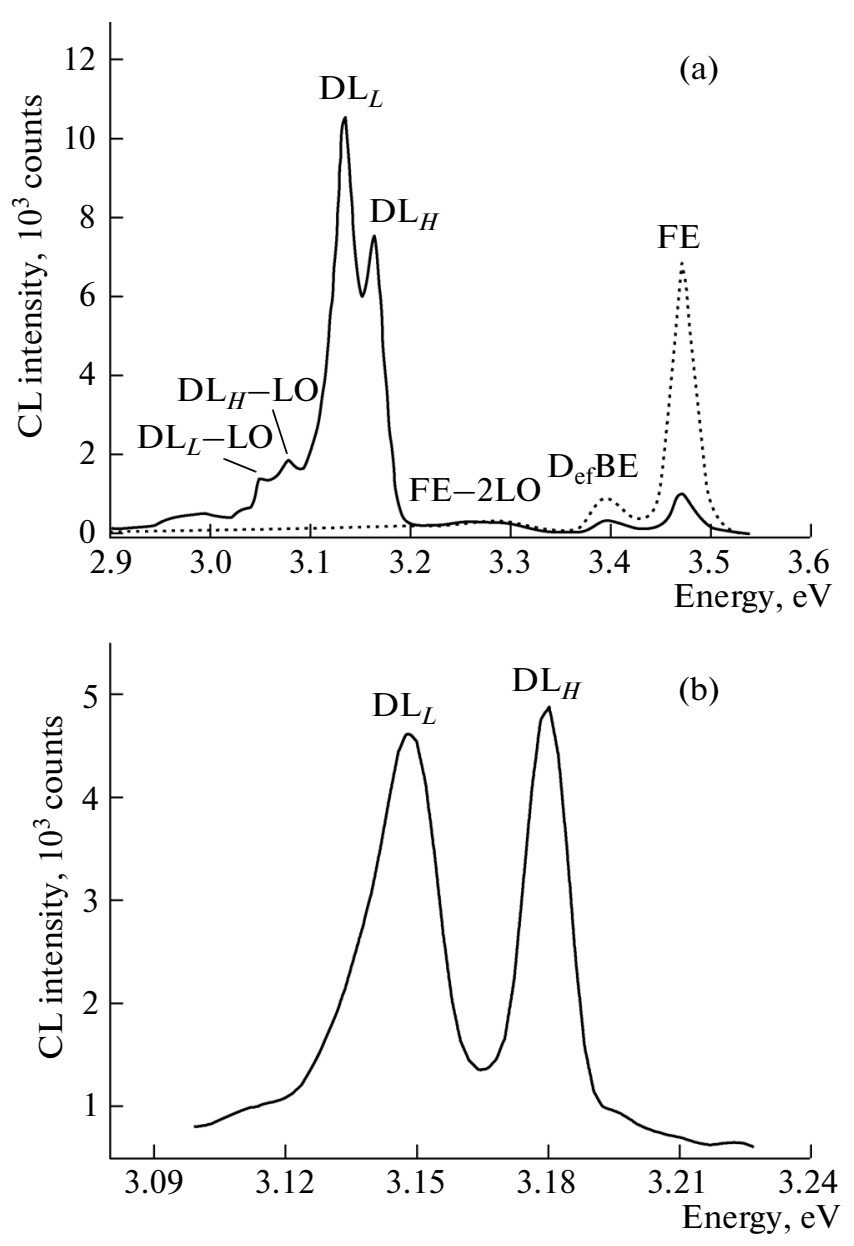

Fig. 2. Cathodoluminescence (CL) spectra measured for immobile electron-beam positions (a) in the region with high dislocation density near the indent (solid curve) and far from it (dashed curve) and (b) in the region of a single screw dislocation. $\mathrm{V}_{a}=10 \mathrm{kV}, I_{\text {beam }}=0.5 \mathrm{nA}, T=70 \mathrm{~K}$.

are efficient sources of intrinsic luminescence with an emission energy $\sim 0.3 \mathrm{eV}$ lower than the band gap.

Generally speaking, the origin of DL with an emission energy lower than the band gap and localized along a dislocation line can be associated with the local electronic states of a perfect dislocation core, partial dislocation cores, stacking faults of splitting dislocation, and point defects accompanying dislocation motion and concentrated near its core.

The rapid motion of fresh luminous dislocations, detected in the experiments presented here, rules out the possibility of explaining the origin of DL by pointdefect traces left by a moving dislocation, since the mechanism of their rapid production and elimination remains unclear in this case. At the same time, the involvement of point defects in the formation of the observed DL band cannot be completely ruled out. For example, in [19], it was shown that a DL band close in spectral position to that detected in the present study disappeared after annealing at $500^{\circ} \mathrm{C}$; at 

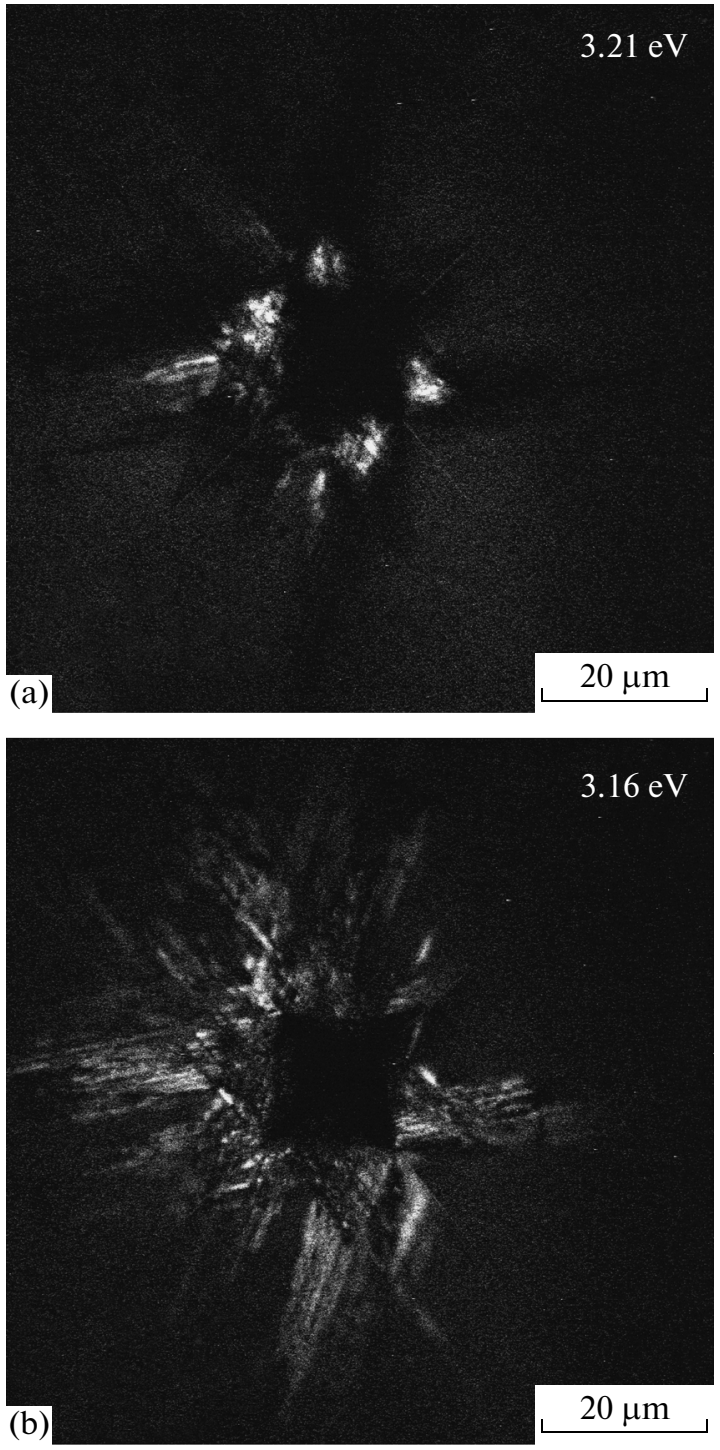

Fig. 3. Cathodoluminescence image of dislocations in the monochromatic mode. The mapping wavelengths are (a) 388 and (b) $394 \mathrm{~nm} . V_{a}=10 \mathrm{kV}, I_{\text {beam }}=0.5 \mathrm{nA}, T=70 \mathrm{~K}$

the same time, a similar luminescence band designated in the review [20] as $Y_{7}$ was observed by many researchers [21-24] in undeformed samples and was attributed to point defects near grown-in $a$-dislocations.

Theoretical calculations of the energy position of the electronic states of dislocations themselves predict the existence of two groups of such states: shallow delocalized states of the deformation potential and deep states concentrated directly in the core.

The core-state depth estimated for an undissociated 60-degree dislocation with a large inaccuracy of $0.2 \mathrm{eV}$ [25] is also too small, and the depth of the core states of partial 30- and 90-degree dislocations, calculated in [26], appears too large $(0.6-1.1 \mathrm{eV})$ in comparison with the data presented.
Perfect screw dislocations create only shear lattice strains which, according to symmetry considerations, lead to the formation of shallow bound states only at the top of the valence band with energies of $<100 \mathrm{meV}$ in direct-gap semiconductors [27, 28]. Recent calculations [15] considering the contributions of higher bands predicted also the existence of an attractive potential for electrons in the conduction band near perfect screw dislocations; this was used in [15] to explain a shift of the DL spectral band by $0.14 \mathrm{eV}$ with respect to the exciton line, but does not allow explanation of a similar shift by $0.3 \mathrm{eV}$ in our case.

Based on the latter, it is reasonable to assume that the additional DL-band shift by $0.15 \mathrm{eV}$ in our samples in comparison with the DL band in [15] is caused by a difference in the structure of the screw-dislocation core, which, in turn, is caused by different doping levels of materials. In [15], high-resistance iron-doped GaN samples with perfect screw dislocations were studied. In the experiments presented here, the samples were low-resistance and not intentionally doped; therefore, it is reasonable to assume that their screw dislocations were dissociated. This assumption is based on electron-microscopy observations of splitted $a$-screw dislocations with a SF width of $5 \mathrm{~nm}$ in undoped gallium nitride [29] and on the well-known effect of the doping level (Fermi-level position) on dislocation splitting in silicon carbide [30, 31], i.e., a phenomenon which, to our knowledge, has not yet been observed in $\mathrm{GaN}$.

The split core of $a$-screw dislocations contains a SF bounded by two 30 -degree dislocations. According to the proposed model [32], dislocation-core splitting is caused by a gain in the total crystal energy when electrons are trapped at deep SF states. In the wurtzite lattice, the SF can be presented to first approximation as a unit-interlayer inclusion of the cubic phase which in both $\mathrm{GaN}$ and $\mathrm{SiC}$ has a smaller band gap than in the hexagonal matrix, mainly due to the conduction-band step. In GaN, the wurtzite- and cubic-phase band gaps are $3.47 \mathrm{eV}$ and $3.27 \mathrm{eV}$, respectively [33]. Theoretical calculations [34] confirm the presence of a type-II heterostructure at the wurtzite-sphalerite interface in $\mathrm{GaN}$; it has long been proposed that SFs be considered as quantum wells in this material [35]. In this case, the additional red-shift of the DL band of an extended dislocation in comparison with a perfect dislocation can be explained by the fact that the electron size-quantization level of the SF, which lies deeper than the electronic level of the deformation potential for an undissociated dislocation, is involved in the optical transition.

The luminescence of different SF types in GaN has been studied in considerable detail [36-38]. According to the data obtained, three stacking faults existing in wurtzite, usually denoted as $I_{1}, I_{2}, E$, yield emission lines differing in energy in the range from 3.29 to $3.42 \mathrm{eV}$ [36]. Only one of these three SF types, i.e., $I_{2}$, 
can arise due to simple gliding in the basal plane and be a component of moving dislocations. According to different data, the energy position of the corresponding luminescence line is in the range of $3.32-3.36 \mathrm{eV}$, i.e., is shifted with respect to the exciton energy exactly by a value of $0.15 \mathrm{eV}$, which is necessary to explain the difference in the DL spectra of samples with different resistivities.

It is impossible to unambiguously determine the origin of the fine doublet structure of the DL spectrum based on the data obtained. In the proposed model, one of the possible explanations, also by analogy with $\mathrm{SiC}$ [5], is associated with the shallow-hole-level optical transitions of two partial dislocations which differ in the atomic composition of their cores: the cores of one of the 30-degree dislocations mainly consist of gallium atoms, and the cores of the other consist of nitrogen atoms. At the same time, the effect of the dislocation splitting width on the DL spectral position in Ge is well known [2]. The mechanism responsible for the DL doublet structure in the case at hand has yet to be clarified.

\section{CONCLUSIONS}

It was shown that $a$-screw dislocations introduced by plastic deformation at room temperature by $\mathrm{GaN}$ indentation or scratching are efficient sources of intrinsic luminescence in the spectral range of 380$390 \mathrm{~nm}$ with a higher probability of optical transitions than in the case of the bulk exciton. Only perfect long straight segments of $a$-screw dislocations are luminous, which can move upon excitation with an electron beam and at a temperature of $70 \mathrm{~K}$. The emission band of individual screw dislocations has a small spectral width comparable to the band widths of free and bound excitons and the doublet structure with a splitting of $\sim 30 \mathrm{meV}$ independent of external strain.

A model was proposed to explain the DL as optical transitions between the electron size-quantization level of the stacking fault and hole levels of two partial 30 -degree dislocations bounding it in the split core of a $a$-screw dislocation.

\section{ACKNOWLEDGMENTS}

We are grateful to Yu.G. Shreter and V.V. Voronenkov for putting the gallium-nitride crystals at our disposal and for stimulating discussions. CL measurements were performed using equipment of the Interdisciplinary Resource Center for Nanotechnology of Saint Petersburg State University.

This study was supported in part by the Optek Company, grant no. 2/201417.

\section{REFERENCES}

1. E. A. Steinman and H. G. Grimmeiss, Semicond. Sci. Technol. 13, 124 (1998).
2. A. N. Izotov, A. I. Kolyubakin, S. A. Shevchenko, and E. A. Steinman, Phys. Status Solidi A 130, 193 (1992).

3. V. D. Negrii, J. Appl. Phys. 74, 7008 (1993).

4. O. Vyvenko and A. Zozime, Mater. Sci. Eng. B 24, 105 (1994).

5. H. Rii, T. Hidekazu, T. Michio, M. I. Kohei, and M. Koji, Appl. Phys. Express 6, 011301 (2013).

6. Y. G. Shreter, Y. T. Rebane, T. J. Davis, J. Barnard, M. Darbyshire, J. W. Steeds, W. G. Perry, M. D. Bremser, and R. F. Davis, Mater. Res. Soc. 449, 683 (1997).

7. M. Albrecht, A. Cremades, J. Krinke, S. Christiansen, O. Ambacher, J. Piqueras, H. P. Strunk, and M. Stutzmann, Phys. Status Solidi B 216, 409 (1999).

8. M. Albrecht, J. L. Weyher, B. Lucznik, I. Grzegory, and S. Porowski, Appl. Phys. Lett. 92, 231909 (2008).

9. I. Ratschinski, H. S. Leipner, F. Heyroth, W. Fränzel, O. Moutanabbir, R. Hammer, and M. Jurisch, J. Phys.: Conf. Ser. 281, 012007 (2011).

10. J. Huang, K. Xu, X. J. Gong, J. F. Wang, Y. M. Fan, J. Q. Liu, X. H. Zeng, G. Q. Ren, T. F. Zhou, and H. Yang, Appl. Phys. Lett. 98, 1 (2011).

11. M. A. Reshchikov and H. Morkoç, J. Appl. Phys. 97, 061301 (2005).

12. I. Yonenaga, Y. Ohno, T. Yao, and K. Edagawa, J. Cryst. Growth 403, 72 (2014).

13. M. Albrecht, H. P. Strunk, J. L. Weyher, I. Grzegory, S. Porowski, and T. Wosinski, J. Appl. Phys. 92, 2000 (2002).

14. O. Medvedev, O. Vyvenko, A. Bondarenko, and V. Voronenkov, in Proceedings of the 12th International Workshop on Beam Injection Assessment of Microstructures in Semiconductors-BIAMS 12 (Tsukuba, Japan, 2014), p. MoA4.

15. M. Albrecht, L. Lymperakis, and O. Neugebauer, Phys. Rev. B 241201, 1 (2014).

16. V. Voronenkov, N. Bochkareva, R. Gorbunov, P. Latyshev, Y. Lelikov, Y. Rebane, A. Tsyuk, A. Zubrilov, and Y. Shreter, Jpn. J. Appl. Phys. 52, 08JE14 (2013).

17. J. Huang, K. Xu, X. J. Gong, J. F. Wang, Y. M. Fan, J. Q. Liu, X. H. Zeng, G. Q. Ren, T. F. Zhou, and H. Yang, Appl. Phys. Lett. 98, 221906 (2011).

18. I. Ratschinski, H. S. Leipner, F. Heyroth, W. Fränzel, O. Moutanabbir, R. Hammer, and M. Jurisch, J. Phys.: Conf. Ser. 281, 012007 (2011).

19. J. Huang, K. Xu, Y. M. Fan, J. F. Wang, J. C. Zhang, and G. Q. Ren, Nanoscale Res. Lett. 9, 649 (2014).

20. M. A. Reshchikov and H. Morkoç, J. Appl. Phys. 97, 061301 (2005).

21. G. Salviati, M. Albrecht, C. Zanotti-Fregonara, N. Armani, M. Mayer, Y. Shreter, M. Guzzi, Y. V. Melnik, K. Vassilevski, V. A. Dmitriev, and H. P. Strunk, Phys. Status Solidi A 171, 325 (1999).

22. E. Calleja, M. A. Sanchez-Garcia, F. J. Sanchez, F. Calle, F. B. Naranjo, E. Munoz, U. Jahn, and K. Ploog, Phys. Rev. B 62, 826 (2000).

\section{$\begin{array}{llll}\text { SEMICONDUCTORS Vol. } 49 & \text { No. } 9 & 2015\end{array}$}


23. F. Bertram, J. Christen, M. Schmidt, M. Topf, S. Koymov, S. Fischer, and B. Meyer, Mater. Sci. Eng. B 50, 165 (1997).

24. N. Grandjean, M. Leroux, M. Laügt, and J. Massies, Appl. Phys. Lett. 71, 240 (1997).

25. A. T. Blumenau, C. J. Fall, J. Elsner, R. Jones, M. I. Heggie, and T. Frauenheim, Phys. Status Solidi C 1709, 1684 (2003).

26. G. Savini, A. T. Blumenau, M. I. Heggie, and S. Öberg, Phys. Status Solidi C 4, 2945 (2007).

27. J. L. Farvacque and P. Franc, Phys. B 274, 995 (1999).

28. Y. T. Rebane and J. W. Steeds, Phys. Rev. B 48, 963 (1993).

29. D. Zakharov, Z. Liliental-Weber, B. Wagner, Z. Reitmeier, E. Preble, and R. Davis, Phys. Rev. B 71, 235334 (2005).

30. A. Galeckas, J. Linnros, and P. Pirouz, Phys. Rev. Lett. 96, 025502 (2006).

31. J. D. Caldwell, R. E. Stahlbush, M. G. Ancona, O. J. Glembocki, and K. D. Hobart, J. Appl. Phys. 108, 044503 (2010).
32. P. Pirouz, Phys. Status Solidi A 210, 181 (2013).

33. J. Menniger, U. Jahn, O. Brandt, H. Yang, and K. Ploog, Appl. Phys. Lett. 69, 836 (1996).

34. C. Stampfl, C. van de Walle, Phys. Rev. B 57, R15052 (1998).

35. Y. T. Rebane, Y. G. Shreter, and M. Albrecht, Phys. Status Solidi A 164, 141 (1997).

36. J. Lähnemann, U. Jahn, O. Brandt, T. Flissikowski, P. Dogan, and H. T. Grahn, J. Phys. D: Appl. Phys. 47, 423001 (2014).

37. R. Liu, A. Bell, F. A. Ponce, C. Q. Chen, J. W. Yang, and M. A. Khan, Appl. Phys. Lett. 86, 021908 (2005).

38. I. Tischer, M. Feneberg, M. Schirra, H. Yacoub, R. Sauer, K. Thonke, T. Wunderer, F. Scholz, L. Dieterle, E. Müller, and D. Gerthsen, Phys. Rev. B 83, 035314 (2011).

Translated by A. Kazantsev 